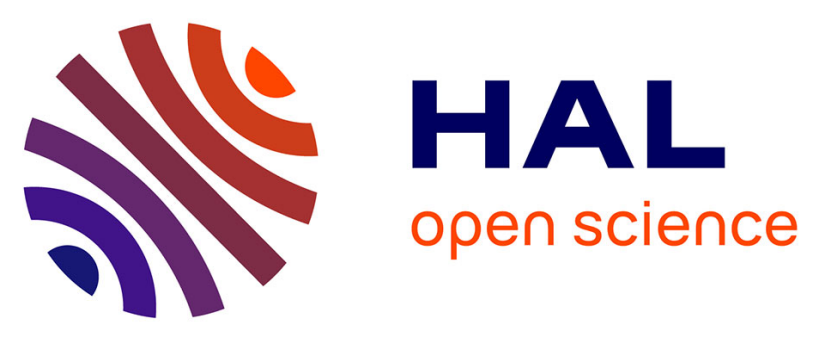

\title{
On the influence of the shoulder kinematic chain on joint kinematics and musculotendon lengths during wheelchair propulsion estimated from multibody kinematics optimization
}

Pierre Puchaud, Samuel Hybois, Antoine Lombart, Joseph Bascou, Hélène

Pillet, Pascale Fodé, Christophe Sauret

\section{To cite this version:}

Pierre Puchaud, Samuel Hybois, Antoine Lombart, Joseph Bascou, Hélène Pillet, et al.. On the influence of the shoulder kinematic chain on joint kinematics and musculotendon lengths during wheelchair propulsion estimated from multibody kinematics optimization. Journal of Biomechanical Engineering, 2019, 141 (10), pp.1-19. 10.1115/1.4043441 . hal-02530750

\author{
HAL Id: hal-02530750 \\ https://hal.science/hal-02530750
}

Submitted on 3 Apr 2020

HAL is a multi-disciplinary open access archive for the deposit and dissemination of scientific research documents, whether they are published or not. The documents may come from teaching and research institutions in France or abroad, or from public or private research centers.
L'archive ouverte pluridisciplinaire $\mathbf{H A L}$, est destinée au dépôt et à la diffusion de documents scientifiques de niveau recherche, publiés ou non, émanant des établissements d'enseignement et de recherche français ou étrangers, des laboratoires publics ou privés. 


\title{
On the influence of the shoulder kinematic chain on joint kinematics and musculotendon lengths during wheelchair propulsion estimated from multibody kinematics optimization
}

\author{
Puchaud Pierre ${ }^{1,2}$, Hybois Samuel ${ }^{1}$, Lombart Antoine ${ }^{1,2}$, Bascou Joseph ${ }^{1,2}$, Pillet Hélène ${ }^{1}$, Fodé \\ Pascale $^{2}$, Sauret Christophe ${ }^{1}$ \\ ${ }^{1}$ Institut de Biomécanique Humaine Georges Charpak, Arts et Métiers ParisTech, 151 Boulevard de l'hôpital, \\ 75013 Paris, France \\ ${ }^{2}$ Centre d'Études et de Recherche sur l'Appareillage des Handicapés, Institution Nationale des Invalides, 47 Rue \\ de l'Echat, 94000 Créteil, France
}

\begin{abstract}
Multibody kinematic optimization is frequently used to assess shoulder kinematics during manual wheelchair (MWC) propulsion but multiple kinematics chains are available. It is hypothesized that these different kinematic chains affect marker tracking, shoulder kinematics and resulting musculotendon (MT) lengths. In this study, shoulder kinematics and MT lengths obtained from four shoulder kinematic chains (open-loop thorax-clavicle-scapula-humerus (M1), closed-loop with contact ellipsoid (M2), scapula rhythm from regression equations (M3), and a single ball-and- socket joint between the thorax and the humerus (M4) were compared. Right-side shoulder kinematics from seven subjects were obtained with 34 reflective markers and a scapula locator using an optoelectronic motion capture system while propelling on a MWC simulator. Data was processed based on the four models. Results showed the impact of shoulder kinematic chains on all studied variables. Marker reconstruction errors were found similar between M1 and M2 and lower than for M3 and M4. Few degrees of freedom (DoF) were noticeably different between M1 and M2, but all shoulder DoFs were significantly affected between M1 and M4. As a consequence of differences in joint kinematics, MT lengths were affected by the kinematic chain definition. The contact ellipsoid (M2) was found as a good trade-off between marker tracking and penetration avoidance of the scapula. The regression-based model (M3) was less efficient due to limited humerus elevation during MWC propulsion, as well as the ball-and-socket model (M4) which appeared not suitable for upper limbs activities, including MWC propulsion.
\end{abstract}

\section{NOMENCLATURE}

$\begin{array}{ll}\text { ACJ } & \text { acromioclavicular joint } \\ \text { DoF } & \text { degree of freedom } \\ \text { GHJ } & \text { glenohumeral joint } \\ \text { MKO } & \text { multibody kinematic optimization } \\ \text { MWC } & \text { manual wheelchair } \\ \text { RMSE } & \text { root mean square error } \\ \text { SCJ } & \text { sternoclavicular joint } \\ \text { STA } & \text { soft tissue artefact } \\ \text { STJ } & \text { scapulothoracic joint } \\ \text { THJ } & \text { thoraco-humeral joint } \\ \text { WAD } & \text { weighted average distance }\end{array}$

\section{INTRODUCTION}

A manual wheelchair (MWC) allows people with walking disabilities to recover a part of their autonomy. However, it has been reported as a constraining form of locomotion inducing upperlimbs pain and injuries [1,2], especially located at the shoulder [3-5]. Musculoskeletal models are often used [6-8]to assess shoulder kinematics and kinetics during MWC propulsion. Such models are tied to the definition of a kinematic chain, which consists of an assembly of rigid bodies - representing bony segments - connected by perfect mechanical joints - such as hinge, 
universal or ball joints - that constrains the motion space. On such kinematic chains, virtual markers located on anatomical landmarks are placed in order to perform a multibody kinematics optimization (MKO, also known as inverse kinematics) $[9,10]$. This MKO process aims at minimizing the root mean square error (RMSE) between experimental and virtual marker positions, to obtain joints kinematics. The main advantage of this technique, with respect to conventional segment kinematics reconstruction, is to avoid joints dislocation and to compensate soft-tissue artefacts. Indeed, segmental kinematics cannot be performed to investigate the shoulder region [11] because of the large soft tissue artifacts of scapula [12,13]. Still, MKO does not necessarily solve the issues linked to motion reconstruction, because to be efficient, it requires a kinematic chain that properly represents joints mobility.

Anatomically, the shoulder is a complex structure including several joints: sternoclavicular (STJ), acromioclavicular (ACJ), scapulothoracic (STJ) and glenohumeral joints (GHJ). Among the models available in the literature, the model of Holzbaur et al. [14,15] and the Delft Shoulder and Elbow model (DSEM) $[6,16]$ were commonly used to investigate MWC propulsion [7,8,17]. The model proposed by Holzbaur [14] relied on coupling regression equations to describe the motion of the scapula and the clavicle from the humerus orientation [18]. Three rotational degrees of freedom (DoF) are defined at the GHJ, two rotational DoF at the elbow and two rotational DoF at the wrist. The DSEM $[6,16]$ was characterized by three rotational DoF for the STJ, ACJ and GHJ and by a gliding contact plane to represent the STJ, which constrained the medial border of the scapula to be in contact with the thorax, modeled by an ellipsoid. Still, modeling the STJ remained a great challenge, because of its non-usual contact type $[12,19]$. Another model, with an ellipsoid mobilizer to prescribe the STJ motion [20], was proposed by Seth et al. [21].
This model is close to the DSEM $[6,16]$, and announced with a low computational cost. It could therefore be suitable for shoulder kinematics assessment during MWC propulsion. Besides, musculoskeletal models associate a muscle set to their kinematic chain to perform musculoskeletal analysis. Thus, changes of musculotendon lengths during a task directly depend on both the muscle set and the kinematic chain. However, the equivalence of the different kinematic chains used in previous MWC studies on joint kinematics and musculotendon lengths remains to be demonstrated.

In this objective, four musculoskeletal models relying on shoulder kinematic chains previously used to study MWC propulsion were implemented to process the same data set. The four shoulder kinematics chains were: open-loop (thorax $\rightarrow$ clavicle $\rightarrow$ scapula $\rightarrow$ humerus), closed-loop with an ellipsoid mobilizer for the STJ [21], open-loop with coupled orientations for clavicle, scapula and humerus [14] and a single ball-joint between the humerus and the thorax. In this study, we aimed at testing the hypothesis of equivalence of these different models on marker reconstruction errors, shoulder joint kinematics and musculotendon lengths.

\section{MATERIALS AND METHODS}

\section{Subject information}

After the approval by the relevant ethics committee (CPP Paris VI Pitié Salpêtrière, France no. 2014-A01203-44), seven subjects (2 females, 5 males) with various levels of disability (poliomyelitis, spinal amyotrophy, congenital malformation, lower limb amputation, or paraplegia) were recruited in the study. Their characteristics were: age: 33.9 years old (SD: 7.9 y.o.; range: 24-46 y.o); height: $1.66 \mathrm{~m}$ (SD: 0.12 $\mathrm{m}$; range: $1.48-1.80 \mathrm{~m}$ ); mass: $66.4 \mathrm{~kg}$ (SD: 9.5 $\mathrm{kg}$; range: $49-79 \mathrm{~kg}$ ) and body mass index: 23.8 $\mathrm{kg} / \mathrm{m}^{2}$ (SD: $1.8 \mathrm{~kg} / \mathrm{m}^{2}$; range: $21.7-26.7 \mathrm{~kg} / \mathrm{m}^{2}$ ). 
Inclusion criteria were to be using a MWC for at least one year and to report no upper-limb pain or injury at the time of the experiments. All the subjects were previously informed of the protocol and gave their written informed consent before the beginning of the experiments.

\section{Experiments and data collection}

Subjects propelled a handrim MWC simulator (Fig. 1) with a friction resistance applied on tires and self-appreciated by the subject to be close to actual displacements. All the subjects propelled on the same MWC simulator. Two acquisitions were performed: subjects were first asked to remain static with hands on knees and then to perform five propulsion cycles at a self-selected comfortable pace.

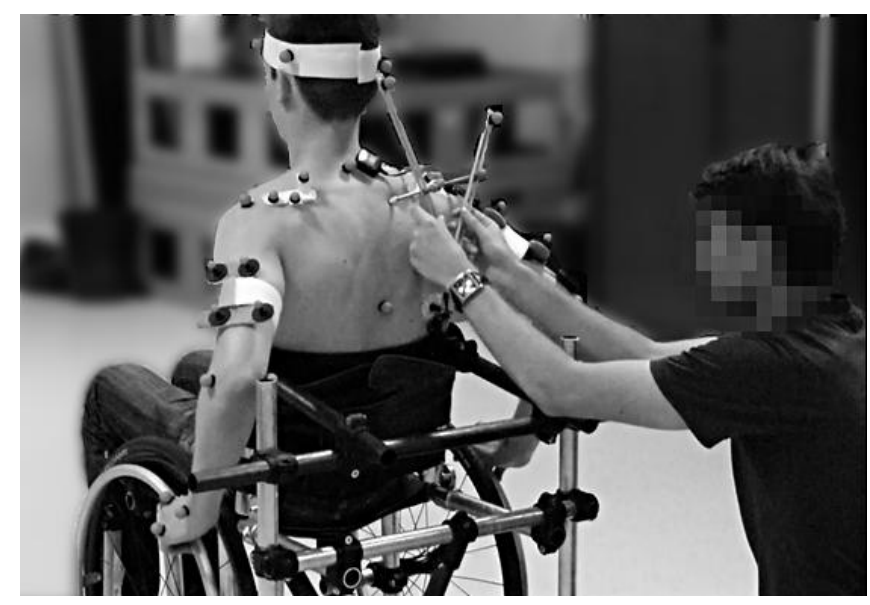

Figure 1: Photograph of a trained experimenter maintaining the scapula locator on the scapula while the subject is propelling on the MWC simulator.

During the experiments, subjects were equipped with 34 skin reflective markers placed on the torso, namely on the manubrium and xyphoid process, and on both spinous processes of the seventh cervical and the eighth thoracic vertebrae; on the head, on the left and right occiput and temporal bones; and on both upper limbs, namely on acromion, lateral and medial epicondyles of the humerus, ulna and radius styloid processes, second and fifth metacarpal heads; following the recommendations of the
International Society of Biomechanics [22]. Moreover, technical markers were placed: a rigid plate with four markers on the humerus, a marker in the middle of the clavicle and another technical marker on the radius to facilitate marker labeling. Furthermore, to minimize soft tissue artifacts in the scapular region, the right scapula was tracked dynamically by a scapula locator (Fig. 1) [23,24] held by a trained experimenter and based on three palpated anatomical landmarks: angulus acromialis, trigonum scapulae and margo medialis. For that purpose, the experimenter performed multiple training trials to adjust the tracking to the specific motion of the scapula according to the task and for each subject. Finally, the three-dimensional locations of the reflective markers were recorded at $100 \mathrm{~Hz}$ using an 8-cameras optoelectronic motion capture system (Vicon® System, COxford Metrics Inc., UK).

\begin{tabular}{lcccc}
\hline Models & STJ & ACJ & STJ & GHJ \\
\hline $\mathbf{M}_{\mathbf{1}}$ & 2 & 3 & No & 3 \\
$\mathbf{M}_{\mathbf{2}}$ & 2 & Constrained & 4 & 3 \\
$\mathbf{M}_{\mathbf{3}}$ & Regression & Regression & No & 3 \\
$\mathbf{M}_{\mathbf{4}}$ & Locked & Locked & No & 3
\end{tabular}

Table 1: Degrees of freedom at the shoulder complex for the 4 models used in this study. "No" means no joint is defined in the kinematic chain implying free motion between the concerned bones. "Locked" means the joint is similar to a weld joint, i.e. no motion

\section{Musculoskeletal Models}

Musculoskeletal models with four different kinematic chains were designed and implemented in OpenSim [25]. Details about the DoF of these different models are summarized in Table 1. The first model (M1) was designed based on the model of Holzbaur et al. [14] which was symmetrized and extended with the head-andneck model of Vasavada et al. [26]. The coupling equations for SCJ and ACJ were removed and the model was provided with two rotational DoF at 
the SCJ (clavicle protraction-retraction and elevation-depression) and three rotational DoF at the ACJ (Fig. 2). The other DoF were identical to the initial models $[14,26]$

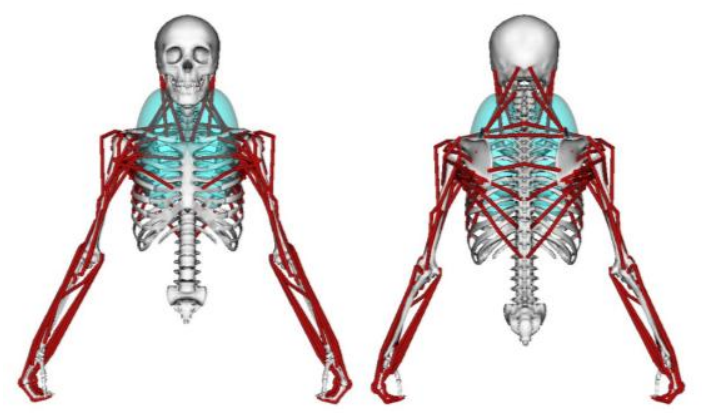

Figure 2: Representation of the musculoskeletal model built in OpenSim for this study. Bone geometries are in white, muscles are in red and ellipsoids are represented in blue. Common muscle sets built for this study is shown.

Based on this first model, three other models were implemented by only modifying the kinematic chain of the shoulder. The second model (M2) defined the STJ with an ellipsoid mobilizer [20,21] and a point constraint between a the ACJ, forming a closed loop. For the third model (M3), the coupling clavicle and scapula motion equations of the Holzbaur model [14,15] were preserved, i.e. regression equations were used to infer the ACJ and STJ rotations from the GHJ elevation [18]. Finally, the fourth model (M4) was implemented by locking both ACJ and SCJ, resulting in only three rotational DoF at the $\mathrm{GHJ}$ for the whole shoulder complex.

To isolate the influence of the kinematic chain on musculotendon lengths, the musculotendon set was the same for all models (Table 2). This set relied on Holzbaur et al. [14] for the upper arm musculotendons and Vasavada et al. [26] for musculotendons involved in scapula and clavicle motions. The remaining musculotendons involved in the shoulder complex, such as rhomboid minor, rhomboid major and serratus anterior were manually added to the model, based on an anatomy atlas [27].

\begin{tabular}{|c|c|c|c|}
\hline Muscles & Abbreviation & $\begin{array}{l}\text { Lines of } \\
\text { action }\end{array}$ & $\begin{array}{l}\text { Adapted } \\
\text { from }\end{array}$ \\
\hline Deltoid & & 3 & {$[14]$} \\
\hline Supraspinatus & DELT & 1 & [14] \\
\hline Infraspinatus & SUPSP & 1 & [14] \\
\hline Subscapularis & INFSP & 1 & [14] \\
\hline Teres Minor & SUPSC & 1 & [14] \\
\hline Teres Major & TMIN & 1 & [14] \\
\hline Pectoralis Major & TMAJ & 3 & [14] \\
\hline Latissimus Dorsi & PECM & 3 & [14] \\
\hline Triceps & LAT & 3 & [14] \\
\hline Aconeous & TRI & 3 & [14] \\
\hline Supinator & $A C$ & 1 & [14] \\
\hline Biceps & SUP & 2 & [14] \\
\hline Brachialis & $\mathrm{BIC}$ & 1 & [14] \\
\hline Brachioradialis & BRA & 1 & [14] \\
\hline Extensor carpi radialis & BRD & 2 & [14] \\
\hline Extensor carpi ulnaris & ECR & 1 & [14] \\
\hline Flexor carpi radialis & ECU & 1 & [14] \\
\hline Flexor carpi ulnaris & FCR & 1 & [14] \\
\hline Pronator Teres & FCU & 1 & [14] \\
\hline Palmaris Longus & PT & 1 & [14] \\
\hline Pronator Quadratus & $\mathrm{PL}$ & 1 & [14] \\
\hline Flexor digitorum & $P Q$ & 2 & [14] \\
\hline superficialis & FDS & 3 & [14] \\
\hline Extensor digitorum & EDC & 3 & [26] \\
\hline communis & SM & 2 & {$[26]$} \\
\hline Sternocleidomastoid & TP & 1 & [26] \\
\hline Trapezius & LS & 2 & [27] \\
\hline Levator scapulae & $\mathrm{RH}$ & 12 & [27] \\
\hline $\begin{array}{l}\text { Rhomboids } \\
\text { Serratus anterior }\end{array}$ & SA & & \\
\hline
\end{tabular}

Table 2: Musculotendon set for the 4 models used in this study. Lines of action are extracted from [14] and [26], or placed anatomically [27].

To keep the same definitions of musculotendon set across the different models, some adaptations were necessary. Some moving path points were thus converted into fixed path points for pectoralis major and latissimus dorsi. This simplification was possible due to the low arm elevations reached during MWC propulsion. Additionally, adaptations were made on a wrapping geometry, below the surgical neck of the humerus, to avoid unrealistic muscle paths and length discontinuities that occurred for MWC propulsion movements, especially at the beginning of the push phase. 


\section{Data processing}

Marker trajectories were smoothed with an average sliding window (5 values). Marker trajectory gaps lower than 15 frames were interpolated using C2-splines. For gaps higher than 15 frames, a registration procedure from the static acquisitions was applied based on singular value decomposition [28].

The beginning of propulsion cycles was automatically identified based on the positive velocity of the barycenter of the four markers placed on the ulna and radius styloid processes, and the second and fifth metacarpal heads. The end of the propulsion cycle was defined by the beginning of a new cycle.

Then, the following process was conducted with OpenSim (Stanford University, 3.3 version) [25], for all models (M1 to M4). First, segments of the four models were scaled to match the dimensions of subjects on the static acquisition, using experimental locations of skin markers and palpated landmarks (using the scapula locator) for the scapula. This was performed with the OpenSim "scaling" tool. Afterwards, MKO was performed $[9,10]$ for propulsion cycles acquisition through the "inverse kinematics" tool, tracking both anatomical, technical and scapula locator markers. Markers placed on both the scapula and the clavicle were not considered for models M3 and M4.

The reconstructed positions and orientations of each segment were expressed in the global coordinate system using the "analyze" tool in Opensim. Homogenous transformation matrices [29] were computed for SCJ, ACJ, STJ, GHJ, as well as the global transformation matrices between the thorax and the humerus. Subsequently, angles were identified with a Y-X'Z" sequence of rotations for SCJ (ProtractionRetraction, Elevation-Depression, InternalExternal Rotation), ACJ (Protraction-Retraction, Posterior-Anterior Tilt, Medial-Lateral Rotation) and STJ (Protraction-Retraction, PosteriorAnterior Tilt, Medial-Lateral Rotation), according to $\mathrm{Wu}$ et. al., [22]. The $Z-X^{\prime}-Y^{\prime \prime}$ sequence of rotations was chosen for $\mathrm{GHJ}$ and the thoracohumeral angles (Flexion-Extension, AdductionAbduction, Internal-External Rotation), as it was reported as more adapted to describe MWC propulsion [30,31].

Finally, musculotendon lengths were computed using OpenSim 3.3 "analyze" tool.

\section{Data Analysis}

The four models were evaluated for marker reconstruction errors, joint kinematics and musculotendon lengths. Average dynamic reconstruction errors between virtual and experimental markers during the propulsion cycles were quantified for the whole marker set through the root mean square error (RMSE) weighted by the assigned weight used during MKO. To refine on the different segments (thorax, clavicle, scapula, and humerus), a weighted average distance (WAD) per segment was also computed [32].

The effect on joint kinematics was evaluated by comparing joint angles and joint center locations. Maximum magnitudes of both joint angles and joint center locations were compared with a Spearman's correlation for which M1 was the reference. M1 was choose as the reference since this model imposed no restriction on the mobility of the scapula. Musculotendon lengths were similarly evaluated through the maximum magnitudes and Spearman's correlation with respect to the results obtained with M1.

\section{Statistical Analysis}

Since normality was not ensured by ShapiroWilk's test, non-parametric Friedman's test ( $p$ value $<0.05$ ) was applied for each segment to demonstrate if the model had an influence on the WAD. Then, Fisher's LSD post-hoc test was 
applied to compare models with one another, on their WAD values.

Also, maximum magnitudes of angles, center of rotation locations and musculotendon lengths were tested through Friedman's test to investigate the influence of the kinematic chain and Fisher's LSD post-hoc was applied to compare models with respect to $\mathrm{M} 1$.

\section{RESULTS}

\section{Marker reconstruction errors}

The mean RMSE obtained with the four models for the whole marker set and the seven subjects are illustrated in Figure 3. For all models, the RMSE was the lowest in the middle of the propulsion cycle (end of the push phase and beginning of the recovery phase). The lowest values were obtained with models $M 1$ and $M 2$. At any time of the propulsion cycle, the standard deviation of the global RMSE was higher for models M3 and M4 than for models M1 and M2. Friedman's test ensured that WAD from different models were different $(p<0.001)$. M1 and M2 exhibited the lowest WAD for all the segments but Fisher's LSD post-hoc did not highlight any significant difference between them (Table 3). As expected, M4 displayed the highest WAD for almost all segments. No significant difference was found between M3 and M4 for all segments WAD.

\section{Joint Kinematics}

Average kinematics of the seven subjects are depicted in Figure 4 for all DoF. By definition, for M4, angles for joint with locked DoF remained constant. This case occurred specifically for the SCJ, STJ and ACJ. SCJ angles exhibited similar time courses for models M1, M2 and M3, but a shift can be observed on SCJ protractionretraction of $\mathrm{M} 2$ with respect to $\mathrm{M} 1$ and $\mathrm{M} 3$. STJ protraction-retraction and mediolateral angles showed the same trend (but shifted) for M1 and $M 2$, but they varied oppositely for $M 3$; which is supported by negative correlation values. STJ anteroposterior tilt remained almost constant for M1 and M2 but varied along the propulsion cycle for M3. The three angles of the ACJ exhibited different patterns and no model seemed to reproduce the same kinematics as M1. The GHJ and thoraco-humeral angles showed similar time courses for M1, M2 and M3, with slight differences in magnitude along the cycle. Even if curve patterns differed between subjects, the same variations between models could be observed. Differences in both flexion-extension and internal-external rotation of GHJ and thoracohumeral joint were observed for M4.

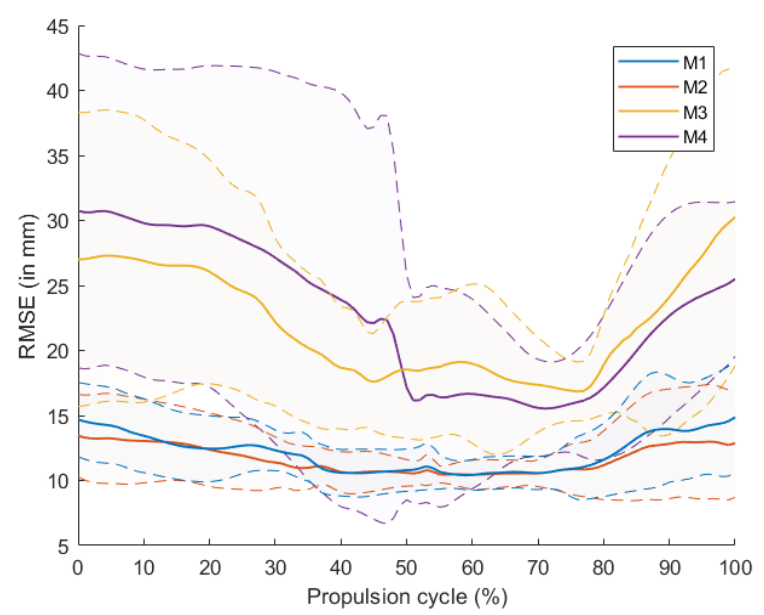

Figure 3: Root mean square error during a propulsion cycle averaged over the 7 subjects: $M 1$ is depicted in blue, M2 in red, M3 in yellow and M4 in purple. Shaded areas represent the standard deviations.

\begin{tabular}{lccccc}
\hline \multirow{2}{*}{ Models Skull } & \multirow{2}{*}{ Thorax } & \multirow{2}{*}{ Clavicle } & Scapula & Humerus \\
\hline \multirow{2}{*}{$\mathbf{M}_{\mathbf{1}}$} & 5.5 & 7.0 & 7.5 & 10 & 13 \\
& {$[3.0-6.7]$} & {$[4.9-8.5]$} & {$[4.4-10]$} & {$[7.8-12]$} & {$[9.6-22]$} \\
$\mathbf{M}_{\mathbf{2}}$ & 4.7 & 7.5 & 7.6 & 9.3 & 14 \\
& {$[2.8-7.0]$} & {$[6.6-9.3]$} & {$[3.8-11]$} & {$[6.4-11]$} & {$[8.3-23]$} \\
$\mathbf{M}_{\mathbf{3}}$ & 8.9 & 14 & 13 & 42 & 18 \\
& {$[5.2-13]^{2}$} & {$[10-17]^{1,2}$} & {$[9.0-17]^{1,2}$} & {$[26-60]^{1,2}$} & {$[10-25]^{1}$} \\
$\mathbf{M}_{4}$ & 10 & 11 & 11 & 32 & 29 \\
& {$[6.4-15]^{1,2}$} & {$[7.8-15]^{1,2}$} & {$[7.2-14]^{1,2}$} & {$[18-40]^{1,2}$} & {$[11-54]^{1,2}$} \\
\hline
\end{tabular}

Table 3: Mean Segment weighted average distance (WAD) and range (in millimeters) for all subjects. Superscripts 1 and 2 indicates the value is significantly different from M1 and $M 2$, respectively $(p<0.05)$. Note that WAD was calculated with the same weights as M1 and M2 for clavicle 
and scapula with M3 and M4 despite they were not considered in the MKO processing.

\begin{tabular}{|c|c|c|c|c|c|c|c|c|}
\hline & \multirow[b]{2}{*}{ Joint Coordinates } & \multirow{2}{*}{$\begin{array}{c}\mathbf{M}_{\mathbf{1}} \\
\text { Magnitude } \\
\text { mean } \pm \text { sd } \\
\left(\text { in }^{\circ}\right)\end{array}$} & \multicolumn{2}{|c|}{$\mathbf{M}_{2}$} & \multicolumn{2}{|c|}{$\mathbf{M}_{3}$} & \multicolumn{2}{|c|}{$\mathrm{M}_{4}$} \\
\hline & & & $\begin{array}{l}\text { Magnitude } \\
\text { mean } \pm \text { sd } \\
\quad\left(\text { in }^{\circ}\right)\end{array}$ & $\begin{array}{l}\text { Correlation } \\
\text { with } \mathrm{M}_{1}\end{array}$ & $\begin{array}{l}\text { Magnitude } \\
\text { mean } \pm \text { sd } \\
\quad\left(\text { in }^{\circ}\right)\end{array}$ & $\begin{array}{c}\text { Correlation } \\
\text { with } \mathrm{M}_{1}\end{array}$ & $\begin{array}{l}\text { Magnitude } \\
\text { mean } \pm \text { sd } \\
\quad\left(\text { in }^{\circ}\right)\end{array}$ & $\begin{array}{c}\text { Correlation } \\
\text { with } \mathrm{M}_{1}\end{array}$ \\
\hline \multirow[t]{2}{*}{$\mathrm{SCJ}$} & Protraction (+)/Retraction (-) ** & $13.1 \pm 2.0$ & $10.6 \pm 1.8$ & $0.93 \pm 0.04$ & $8.8 \pm 2.5$ & $0.85 \pm 0.10$ & $0.0 \pm 0.0^{* * *}$ & $0.01 \pm 0.08$ \\
\hline & Elevation (+)/Depression (-) $* * *$ & $8.2 \pm 2.0$ & $7.4 \pm 2.0$ & $0.83 \pm 0.11$ & $3.2 \pm 0.9^{*}$ & $0.72 \pm 0.24$ & $0.0 \pm 0.0 * * *$ & $0.00 \pm 0.10$ \\
\hline \multirow[t]{3}{*}{ STJ } & Protraction (+)/Retraction (-) $* * *$ & $11.3 \pm 1.4$ & $15.5 \pm 1.9$ & $0.95 \pm 0.01$ & $2.5 \pm 0.8$ & $-0.84 \pm 0.09$ & $0.0 \pm 0.0^{* *}$ & $-0.01 \pm 0.07$ \\
\hline & Posterior (+)/Anterior (-) Tilt ** & $8.0 \pm 2.8$ & $7.7 \pm 2.0$ & $0.73 \pm 0.32$ & $12.2 \pm 3.5$ & $0.68 \pm 0.37$ & $0.0 \pm 0.0^{*}$ & $0.03 \pm 0.08$ \\
\hline & Medial (+)/Lateral (-) Rotation *** & $12.9 \pm 3.1$ & $8.5 \pm 3.2$ & $0.84 \pm 0.25$ & $8.0 \pm 2.2$ & $-0.83 \pm 0.17$ & $0.0 \pm 0.0 * * *$ & $-0.02 \pm 0.03$ \\
\hline \multirow[t]{3}{*}{ ACJ } & Protraction (+)/Retraction (-) $* * *$ & $5.7 \pm 1.7$ & $5.0 \pm 1.9$ & $-0.30 \pm 0.55$ & $8.4 \pm 2.5$ & $0.27 \pm 0.55$ & $0.0 \pm 0.0^{*}$ & $0.02 \pm 0.09$ \\
\hline & Posterior (+)/Anterior (-) Tilt ** & $6.8 \pm 2.5$ & $5.4 \pm 1.9$ & $0.26 \pm 0.63$ & $11.8 \pm 3.4$ & $0.56 \pm 0.46$ & $0.0 \pm 0.0^{*}$ & $-0.01 \pm 0.05$ \\
\hline & Medial (+)/Lateral (-) Rotation *** & $11.3 \pm 2.2$ & $4.7 \pm 2.1^{*}$ & $0.68 \pm 0.37$ & $12.3 \pm 3.4$ & $-0.57 \pm 0.39$ & $0.0 \pm 0.0 * * *$ & $-0.04 \pm 0.10$ \\
\hline \multirow[t]{3}{*}{$\mathrm{GHJ}$} & Flexion $(+) /$ Extension $(-) * * *$ & $44.6 \pm 7.1$ & $47.7 \pm 8.2$ & $0.97 \pm 0.03$ & $\begin{array}{l}60.5 \pm \\
2.7^{* *}\end{array}$ & $0.97 \pm 0.01$ & $\begin{array}{l}103.2 \pm \\
70.5^{* * *}\end{array}$ & $0.73 \pm 0.41$ \\
\hline & Adduction (+)/Abduction (-) & $24.7 \pm 6.9$ & $24.8 \pm 7.1$ & $0.88 \pm 0.17$ & $30.1 \pm 10.5$ & $0.87 \pm 0.11$ & $31.0 \pm 16.5$ & $0.48 \pm 0.56$ \\
\hline & Int (+)/Ext (-) Rotation & $28.8 \pm 8.4$ & $28.7 \pm 8.3$ & $0.97 \pm 0.02$ & $22.6 \pm 3.9$ & $0.93 \pm 0.03$ & $60.4 \pm 76.9$ & $0.66 \pm 0.26$ \\
\hline \multirow[t]{3}{*}{ THJ } & Flexion $(+) /$ Extension $(-)^{* *}$ & $53.3 \pm 6.5$ & $54.6 \pm 6.5$ & $0.99 \pm 0.00$ & $54.8 \pm 4.4$ & $0.97 \pm 0.03$ & $\begin{array}{l}94.4 \pm \\
56.5^{* * *}\end{array}$ & $0.72 \pm 0.48$ \\
\hline & Adduction (+)/Abduction (-) & $30.9 \pm 12.1$ & $33.2 \pm 11.1$ & $0.96 \pm 0.02$ & $27.2 \pm 11.1$ & $0.78 \pm 0.28$ & $34.6 \pm 12.4$ & $0.74 \pm 0.43$ \\
\hline & Int (+)/Ext (-) Rotation & $12.5 \pm 4.4$ & $11.7 \pm 4.7$ & $0.87 \pm 0.07$ & $15.0 \pm 4.9$ & $0.52 \pm 0.45$ & $42.3 \pm 50.6$ & $0.67 \pm 0.32$ \\
\hline
\end{tabular}

Table 4: Mean and standard deviation of joint angle magnitudes in degree: sternoclavicular joint (SCJ), scapulothoracic joint (STJ), acromioclavicular joint (ACJ), glenohumeral joint (GHJ), thoraco-humeral joint (THJ). Significant differences between models are reported in the first column and significant difference with respect to M1 was also reported with *, **, *** indicating respective $p$-values $<0.05,<0.005,<0.001$. Spearman's correlation with M1 is reported for M2, M3 and M4.

\begin{tabular}{|c|c|c|c|c|c|c|c|}
\hline \multirow[b]{2}{*}{ Center of rotation } & \multirow{2}{*}{$\begin{array}{c}\mathbf{M}_{\mathbf{1}} \\
\text { Magnitude } \\
\text { mean } \pm \mathrm{sd} \\
(\text { in } \mathrm{mm})\end{array}$} & \multicolumn{2}{|c|}{$\mathbf{M}_{2}$} & \multicolumn{2}{|c|}{$\mathbf{M}_{3}$} & \multicolumn{2}{|c|}{$\mathbf{M}_{4}$} \\
\hline & & $\begin{array}{l}\text { Magnitude } \\
\text { mean } \pm \mathrm{sd} \\
(\text { in } \mathrm{mm})\end{array}$ & $\begin{array}{c}\text { Correlation } \\
\text { with } \mathrm{M}_{1}\end{array}$ & $\begin{array}{c}\text { Magnitude } \\
\text { mean } \pm \text { sd } \\
(\text { in } \mathrm{mm})\end{array}$ & $\begin{array}{c}\text { Correlation } \\
\text { with } \mathrm{M}_{1}\end{array}$ & $\begin{array}{c}\text { Magnitude } \\
\text { mean } \pm \text { sd } \\
(\text { in } \mathrm{mm})\end{array}$ & $\begin{array}{c}\text { Correlation } \\
\text { with } \mathrm{M}_{1}\end{array}$ \\
\hline $\mathrm{SCJ}$ & $8.9 \pm 5.8$ & $9.4 \pm 5.2$ & $0.78 \pm 0.18$ & $10.5 \pm 3.8$ & $0.29 \pm 0.46$ & $14.0 \pm 6.3$ & $0.04 \pm 0.34$ \\
\hline $\mathrm{ACJ}$ & $24.5 \pm 5.9$ & $22.2 \pm 7.3$ & $0.92 \pm 0.05$ & $21.9 \pm 6.1$ & $0.87 \pm 0.09$ & $18.4 \pm 8.1$ & $0.58 \pm 0.41$ \\
\hline GHJ & $30.2 \pm 7.1$ & $27.4 \pm 8.9$ & $0.95 \pm 0.04$ & $23.2 \pm 7.0$ & $0.91 \pm 0.07$ & $19.4 \pm 8.7$ & $0.54 \pm 0.45$ \\
\hline
\end{tabular}

Table 5: Mean and standard deviation of joint angle magnitudes in degree: sternoclavicular joint (SCJ), scapulothoracic joint (STJ), acromioclavicular joint (ACJ), glenohumeral joint (GHJ), thoraco-humeral joint (THJ). Significant differences between models are reported in the first column and significant difference with respect to M1 was also reported with *, **, *** indicating respective $p$-values $<0.05,<0.005,<0.001$. Spearman's correlation with M1 is reported for M2, M3 and M4. 

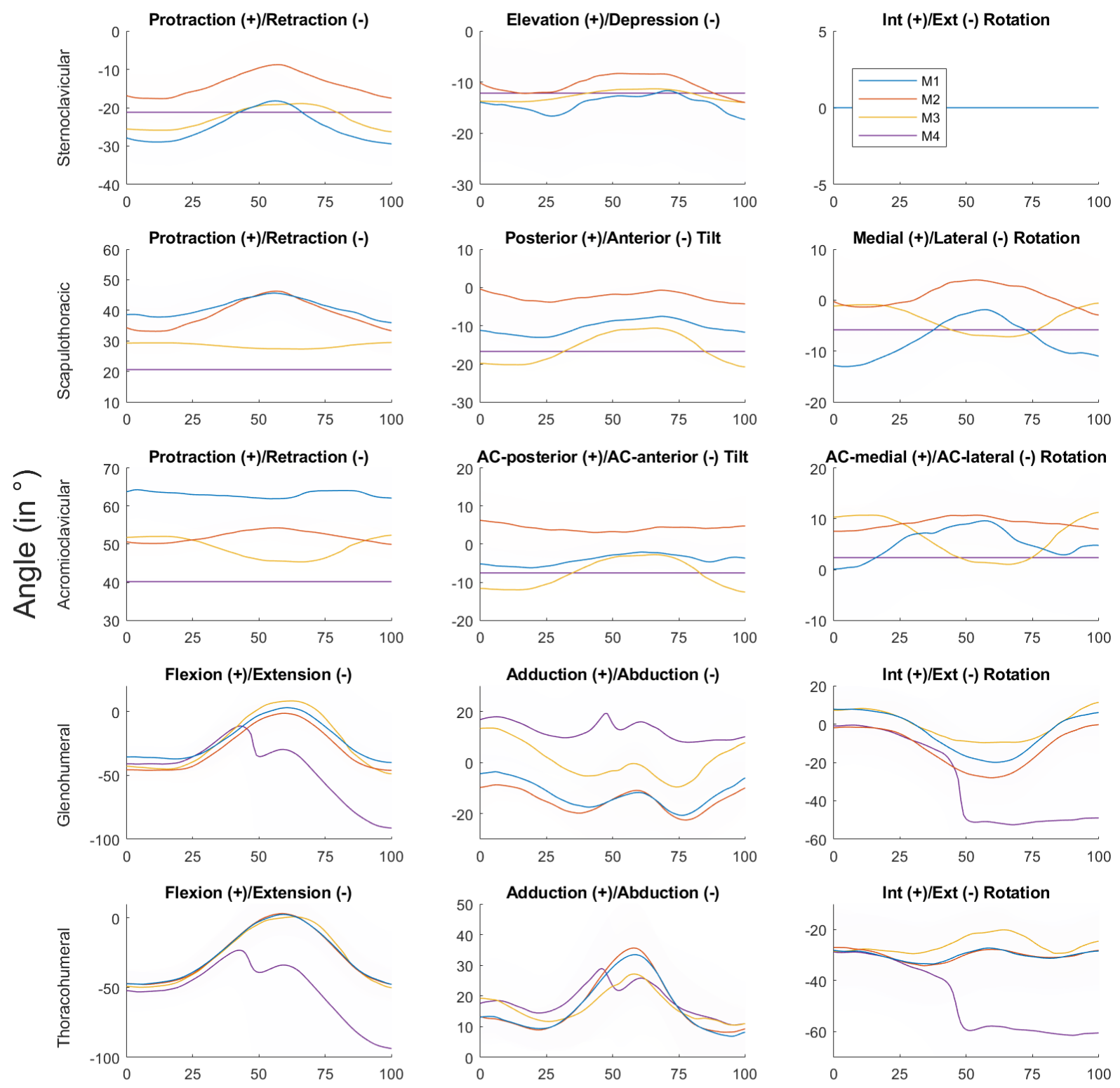

Propulsion cycle (\%)

Figure 4: Mean joint angles: M1 is depicted in blue, M2 in red, M3 in yellow and M4 in purple.

Mean magnitudes of joint angles during the $p<0.001$ ). Significant differences were found for propulsion cycle are reported in Table 4. the joint angle magnitudes of these 10 DoF Comparison between models showed significant between M4 and M1. Significant differences were differences in magnitudes of all SCJ, STJ, and also found in SCJ elevation-depression and GHJ ACJ angles plus GHJ flexion-extension and flexion-extension magnitudes between $\mathrm{M} 3$ and to thoraco-humeral flexion-extension $(p<0.01$ or $\mathrm{M} 1 \quad(p<0.05$ and $p<0.01$, respectively). Finally, 
only a significant difference was found only in magnitude of ACJ mediolateral rotation between $\mathrm{M} 2$ and $\mathrm{M} 1 \quad(\mathrm{p}<0.05)$.

Over the whole population, Spearman's correlations with respect to M1 were greater for M2 and M3 than for M4, for all joints. Correlations were the highest with $M 2$, excepted for ACJ protraction-retraction and posterior-anterior tilt. ACJ protraction-retraction of M2 even demonstrated a negative average of correlations, indicating an inverse relationship between $\mathrm{M} 1$ and M2. Model M2 was overall the closest to M1 for joint kinematics description, especially for GHJ and thoraco-humeral angles. Magnitude of joints centers evolution during the propulsion cycle in the global coordinate system are presented in Table 5. No significant difference in these magnitudes was found between models for every joint. However, Spearman's correlation with respect to $M 1$ was the highest with $M 2$ for both SCJ, ACJ, and GHJ.

\section{Musculotendon Lengths}

Average time courses of the musculotendon lengths over the seven subjects are reported on Figure 5. For most of the musculotendons, the mean evolution along the propulsion cycle was globally resembling between the different models even if some shifts can be observed. The most divergent patterns were obtained for the deltoid middle, the subscapularis and the three lines of action of the latissimus dorsi.

Mean magnitudes of musculotendon lengths during the propulsion cycleare reported in Table 5. Comparison between models showed significant differences in musculotendon length magnitude for anterior, deltoid middle, deltoid posterior, subscapularis, teres minor, pectoralis clavicular, pectoralis sternal and pectoralis rib, latissimus dorsi thoracic and latissimus dorsi lumbar. Significant differences in musculotendon length magnitudes were found between M1 and M4 for deltoid anterior, deltoid middle, deltoid posterior, subscapularis, teres minor, pectoralis sternal and rib. Significant differences in musculotendons length magnitudes were also found between M1 and M3 for deltoid posterior, subscapularis, pectoralis rib, latissimus dorsi thoracic and latissimus dorsi lumbar. Finally, comparison between $\mathrm{M} 1$ and $\mathrm{M} 2$ showed significant differences in musculotendon length magnitudes for deltoid middle, pectoralis clavicular and pectoralis sternal.

For all musculotendon lengths, Spearman's correlations with respect to M1 were greater for M2 and M3 than for M4. Correlations were the highest for $M 2$, excepted for the pectoralis clavicular, which was higher for M3. Depending on the model, mean differences in musculotendon lengths with respect to M1 ranged from 2.6 to 32 $\mathrm{mm}$ for deltoid; from 1.3 to $20 \mathrm{~mm}$ for rotator cuff muscles; from 6.5 to $41 \mathrm{~mm}$ for pectoralis major; from 2 to $31 \mathrm{~mm}$ for triceps longus and biceps longus and up to $91 \mathrm{~mm}$ for latissimus dorsi.

\section{DISCUSSION}

\section{Marker reconstruction errors}

In the present study, the global RMSE was highly reduced with models $\mathrm{M} 1$ and $\mathrm{M} 2$ (about $10-15 \mathrm{~mm}$ all along the propulsion cycle) than with models $M 3$ (17-27 $\mathrm{mm}$ in average during the push phase) and M4 (23-30 $\mathrm{mm}$ in average during the push phase). In addition, the standard deviation of the RMSE was noticeably higher for M3 and M4 than for M1 and M2, which denotes the inability of models $M 3$ and M4 to fit the various kinematics used by the different subjects.

Regarding the literature on upper limb kinematics, marker reconstruction errors associated to MKO was generally reported through the global RMSE for all the markers $[21,33,34]$ or through on a weighted average distance (WAD) per segment [32,34] allowing a more refined analysis. In the present study, the WAD between experimental and reconstructed 

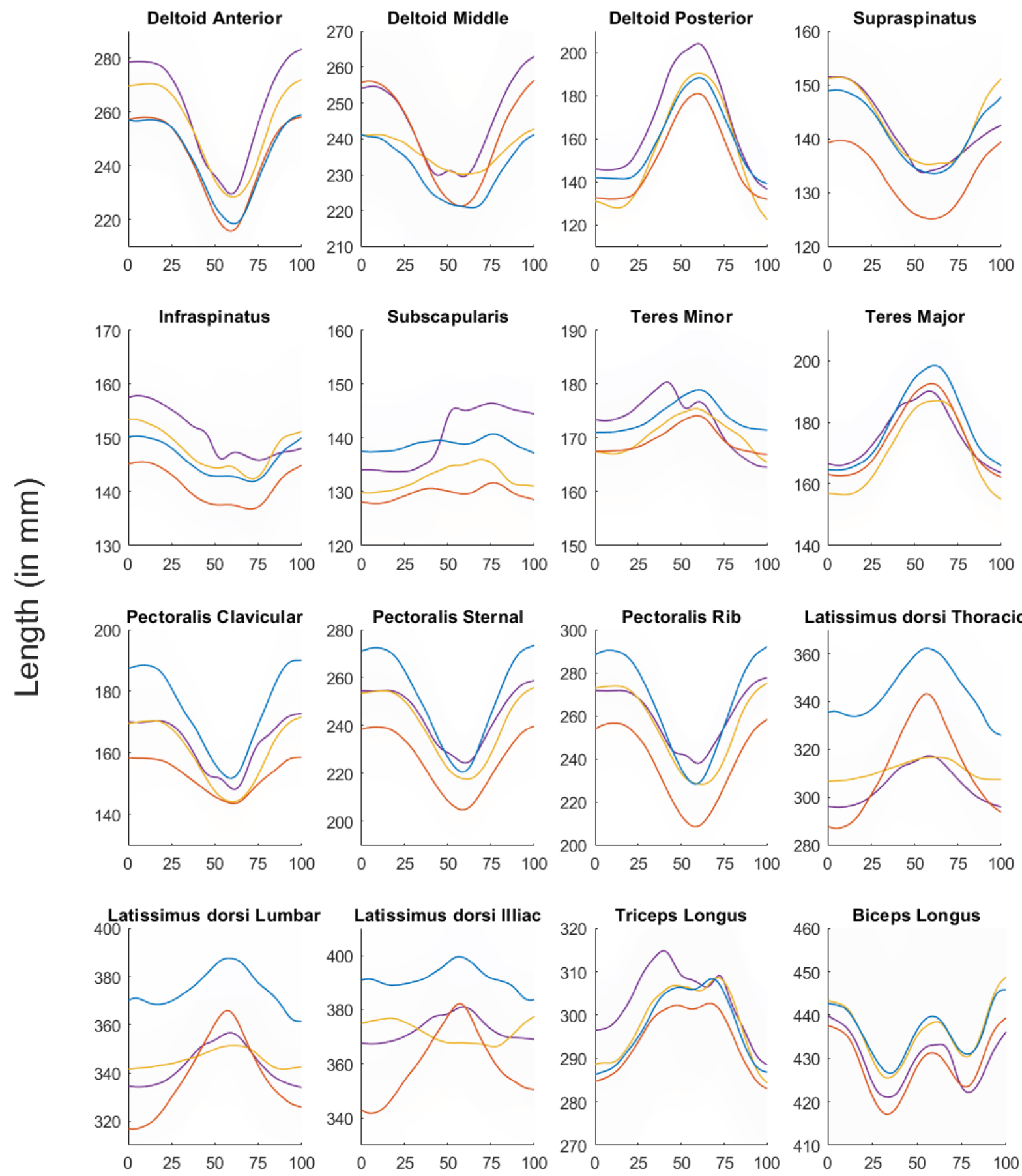

\section{Propulsion cycle (\%)}

Figure 5: Time course of musculotendon lengths of one typical subject for the different models: M1 is depicted in blue, M2 in red, M3 in yellow and M4 in purple. Shaded areas represent the standard deviations. 


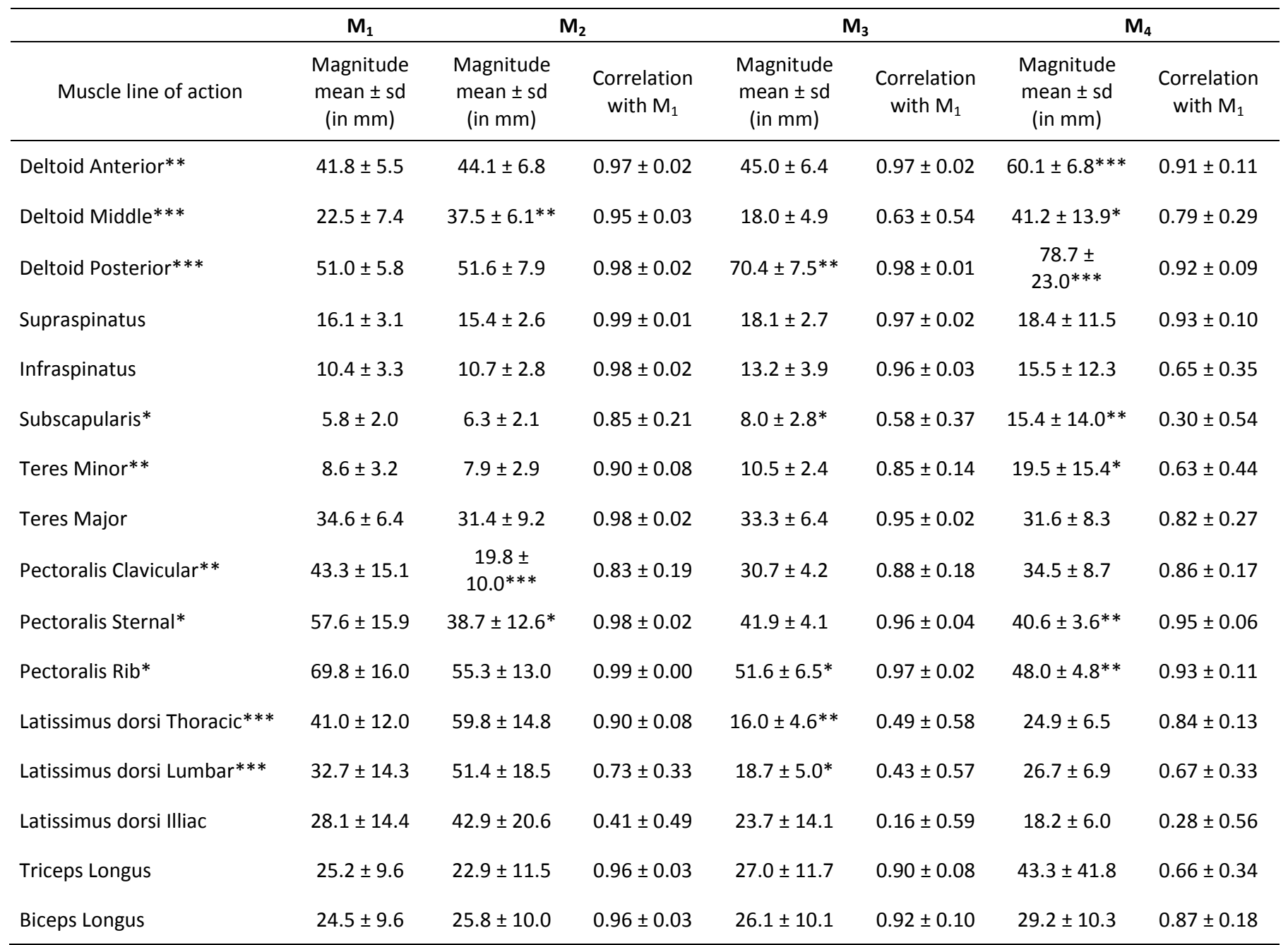

Tableau 6: Mean and standard deviation of musculotendon length magnitudes (in millimeters). Significant differences between models are reported in the first column and significant difference with respect to M1 was also reported with *, **, *** indicating respective p-values $<0.05,<0.005,<0.001$. Spearman's correlation with M1 is reported for M2, M3 and M4.

markers was considered as an indicator of the ability of a model to track the bone motions independently. The underlying assumption was that a better kinematic chain would result in smaller marker reconstruction errors and smaller WAD values. In light of this indicator and among the four tested models, models M1 and M2 did minimize marker reconstruction errors for all segments, i.e. skull, thorax, clavicle, scapula and humerus. Compared to [34] the marker reconstruction errors of the humerus were found slightly higher in our study (4-8 mm vs $8.3-22$ $\mathrm{mm})$. However, this difference may be explained by the personalization of the kinematic chain, which in our study was limited to homothetic ratios for each direction of the segment coordinate system. For the scapula specifically, models M1 and M2 were also reported to be less prone to reconstruction error than models M3 and M4. It is reminded here that the tracking of the scapula during the propulsion was performed through a dynamic tracking with a scapula locator [23]. This technique was used to limit the effect of STA, which can reach up to $80 \mathrm{~mm}$ [35]. The use of this scapula locator resulted in marker reconstruction errors in accordance with results 
obtained with intra-cortical pins for the clavicle, scapula, thorax and skull. Indeed, WAD for the different segment ranged from 2.8 to $12 \mathrm{~mm}$ for M1 and M2, which are equivalent to [33] (5-14 $\mathrm{mm})$. Hence, the use of this scapula locator allowed focusing on the ability of the kinematics chain to reconstruct the bony motions while limiting bias created by STA.

Hence, if M3 and M4 were found suitable enough to fit the shoulder kinematics of some subjects, they can be unable to fit the kinematics of some others. On the opposite, M1 and M2 allow to adapt to the various shoulder kinematics used to propel a MWC. Looking specifically on M1, this model exhibited the lowest reconstruction errors. However, some penetrations of the reconstructed scapula in the thorax were observed with this model. The use of an ellipsoid mobilizer such as in model M2 may prevent from this non-physiological scapula penetration without important losses of accuracy for marker reconstruction. Indeed, reducing the number of DoFs, by defining closed loops did not increase marker reconstruction errors. It may indicate a higher biofidelity of the model M2 [9].

\section{Joint kinematics}

Regarding joint kinematics, this study confirms that joint angles obtained through MKO are affected by the kinematic chain [12,19,34,36], even when studying MWC propulsion. Indeed, non-negligible differences can be observed in all DoF between models. However, M1 and M2 provided joint angles with similar patterns for most of the DoF even if curves were shifted, Figure 3. These offsets can be explained by the constraints imposed in M2 (a closed-loop between the thorax, the clavicle and the scapula), which limited the compensation of inappropriate bodies lengths trough the different joints. In particular, the scaling of the clavicle length was already shown as crucial for the shoulder kinematics obtained with MKO [37]. Effort on ellipsoid parameters identification (radii, center and orientation) should also be made for more reliable shoulder kinematics [38]. Hence, subjectspecific approaches should be considered for this purpose.

In terms of joint angles magnitudes during the propulsion cycle, models $\mathrm{M} 1$ and $\mathrm{M} 2$ provide comparable results for $\mathrm{GHJ}$ and thoraco-humeral angles but differences can be noted in ACJ mediolateral rotation. Low correlations were also obtained for STJ postero-anterior tilt and SCJ angles between these models. Model M3 did not display similar kinematics with respect to $M 1$ and M2 for STJ protraction-retraction and ACJ angles. In this model, the protraction-retraction of the scapula was obtained from the humerus elevation through coupled coordinates. Because of the low humerus elevation during MWC propulsion, this model is unable to provide such scapula motion. Hence, even if extensively used in the MWC literature [39-41], model M3 appeared less suitable compared to M2 to study shoulder kinematics during MWC propulsion.

\section{Musculotendon lengths}

Some studies already reported the drastic effect of joint kinematic variations on computed muscles and joint contact forces [22,33]. Indeed, when computing muscle activations and forces, changes in kinematic parameters play a crucial role because they directly impact musculotendon lengths, shortening/lenghtening velocities and moment arms. To the knowledge of the authors, no study reported the effect of the kinematic chain definition on musculotendon lengths in the specific case of MWC propulsion. Even if models M1 and M2 provided the closest results in terms of markers reconstruction and joint kinematics, differences in musculotendon lengths were found for middle deltoid, pectoralis clavicular, pectoralis sternal, lumbar and iliac latissimus dorsi. Interestingly, M3 resulted in opposite evolution for three subjects with respect to other models for 
deltoid middle and latissimus dorsi. Hence, this study showed the impact of the kinematic chain on musculotendon parameters such as length and shortening/lengthening velocity, which would impact the computed muscle activation determined in musculoskeletal simulation due to the force/length and force/velocity relationships.

\section{Study limitations}

Some limitations can however be considered in this study. In particular, due to the absence of gold standard, it is not possible to strictly conclude on the most suitable model for the determination of bone and joint kinematics. Consequently, the marker reconstruction errors were considered as the criterion for accuracy in bone and joint kinematics with the underlying assumption was that a better kinematic chain would result in smaller marker reconstruction errors. However, considering the level of marker reconstruction errors provided by models $M 3$ and M4, these models can be assumed less suitable than models M1 and M2 to describe the shoulder motion during MWC propulsion. For the same reason, no reference was provided for musculotendon lengths and shortening/lengthening velocities. However, this does not challenge the conclusion of this study on the impact of the kinematic chain on musculotendon parameters.

\section{CONCLUSION}

This study aimed at investigating the effect of four shoulder kinematic chains used to study MWC propulsion on marker reconstruction errors, joint kinematics and musculotendon lengths. These models relied on (1) an open-loop thorax $\rightarrow$ clavicle $\rightarrow$ scapula $\rightarrow$ humerus; (2) a closedloop with a contact ellipsoid between the scapula and the thorax; (3) an open-loop with coupled orientations for clavicle, scapula and humerus; and (4) an open-loop with a single ball joint between the humerus and the thorax. Results showed the non-negligible effect of the shoulder kinematic chain, rejecting the hypothesis of equivalence of these models, on all the studied variables. In particular, modeling the shoulder complex only by the glenohumeral joint appeared less suitable than others models allowing movement of the scapula with respect to the thorax. Coupled coordinates from regression equations to assess the orientations of the scapula and the clavicle[14,15], did not appear to be the most convenient model to compute shoulder kinematics in the study of MWC propulsion. The use of a contact ellipsoid to describe the contact between the scapula and the thorax was found more appropriate since it avoided joint dislocations and body penetrations. However, the personalization of kinematic chains remains to be enhanced, with medical imaging or optimization methods $[42,43]$ to scale the clavicle length [37], and ellipsoid parameters (center, radii and orientations) [38].

\section{ACKNOWLEDGMENT}

The authors hereby affirm that the study does not raise any conflict of interest.

\section{FUNDING}

This study has been self-funded by the Centre d'Etude et de Recherche sur l'Appareillage des Handicapés (Institution Nationale des Invalides), Créteil, France.

\section{REFERENCES}

[1] Boninger, M. L., Dicianno, B. E., Cooper, R. A., Towers, J. D., Koontz, A. M., and Souza, A. L., 2003, "Shoulder Magnetic Resonance Imaging Abnormalities, Wheelchair Propulsion, and Gender1," Arch. Phys. Med. Rehabil., 84(11), pp. 1615-1620.

[2] Mercer, J. L., Boninger, M., Koontz, A., Ren, D., DysonHudson, T., and Cooper, R., 2006, "Shoulder Joint Kinetics and Pathology in Manual Wheelchair Users," Clin. Biomech., 21(8), pp. 781-789. 
[3] Curtis, K. A., Drysdale, G. A., Lanza, R. D., Kolber, M., Vitolo, R. S., and West, R., 1999, "Shoulder Pain in Wheelchair Users with Tetraplegia and Paraplegia," Arch. Phys. Med. Rehabil., 80(4), pp. 453-457.

[4] Finley, M. A., and Rodgers, M. M., 2004, "Prevalence and Identification of Shoulder Pathology in Athletic and Nonathletic Wheelchair Users with Shoulder Pain: A Pilot Study," J. Rehabil. Res. Dev., 41(3B), pp. 395402.

[5] Heyward, O. W., Vegter, R. J. K., Groot, S. de, and Woude, L. H. V. van der, 2017, "Shoulder Complaints in Wheelchair Athletes: A Systematic Review," PLOS ONE, 12(11), p. e0188410.

[6] Blana, D., Hincapie, J. G., Chadwick, E. K., and Kirsch, R. F., 2008, "A Musculoskeletal Model of the Upper Extremity for Use in the Development of Neuroprosthetic Systems," J. Biomech., 41(8), pp. 1714-1721.

[7] Odle, B., Reinbolt, J., Forrest, G., and Dyson-Hudson, T., 2018, "Construction and Evaluation of a Model for Wheelchair Propulsion in an Individual with Tetraplegia," Med. Biol. Eng. Comput.

[8] van Drongelen, S., van der Woude, L. H., Janssen, T. W., Angenot, E. L., Chadwick, E. K., and Veeger, D. H., 2005, "Glenohumeral Contact Forces and Muscle Forces Evaluated in Wheelchair-Related Activities of Daily Living in Able-Bodied Subjects Versus Subjects With Paraplegia and Tetraplegia," Arch. Phys. Med. Rehabil., 86(7), pp. 1434-1440.

[9] Begon, M., Andersen, M. S., and Dumas, R., 2018, "Multibody Kinematics Optimization for the Estimation of Upper and Lower Limb Human Joint Kinematics: A Systematized Methodological Review," J. Biomech. Eng., 140(3), p. 030801.

[10] Lu, T.-W., and O'Connor, J. J., 1998, "Bone Position Estimation from Skin Marker Co-Ordinates Using Global Optimisation with Joint Constraints," J. Biomech., 32(2), pp. 129-134.

[11] Chèze, L., Fregly, B. J., and Dimnet, J., 1995, “A Solidification Procedure to Facilitate Kinematics Analyses Based on Video System Data," J. Biomech., 28(7), pp. 879-884.

[12] Naaim, A., Moissenet, F., Duprey, S., Begon, M., and Chèze, L., 2017, "Effect of Various Upper Limb Multibody Models on Soft Tissue Artefact Correction: A Case Study," J. Biomech., 62, pp. 102-109.

[13] Šenk, M., and Chèze, L., 2010, "A New Method for Motion Capture of the Scapula Using an Optoelectronic Tracking Device: A Feasibility Study," Comput. Methods Biomech. Biomed. Engin., 13(3), pp. 397-401.

[14] Holzbaur, K. R. S., Murray, W. M., and Delp, S. L., 2005, "A Model of the Upper Extremity for Simulating Musculoskeletal Surgery and Analyzing Neuromuscular Control," Ann. Biomed. Eng., 33(6), pp. 829-840.

[15] Saul, K. R., Hu, X., Goehler, C. M., Vidt, M. E., Daly, M., Velisar, A., and Murray, W. M., 2015, "Benchmarking of Dynamic Simulation Predictions in
Two Software Platforms Using an Upper Limb Musculoskeletal Model," Comput. Methods Biomech. Biomed. Engin., 18(13), pp. 1445-1458.

[16] van der Helm, F. C. T., 1994, "A Finite Element Musculoskeletal Model of the Shoulder Mechanism," J. Biomech., 27(5), pp. 551-569.

[17] Slowik, J. S., Requejo, P. S., Mulroy, S. J., and Neptune, R. R., 2016, "The Influence of Wheelchair Propulsion Hand Pattern on Upper Extremity Muscle Power and Stress," J. Biomech., 49(9), pp. 1554-1561.

[18] de Groot, J. H., and Brand, R., 2001, "A ThreeDimensional Regression Model of the Shoulder Rhythm," Clin. Biomech. Bristol Avon, 16(9), pp. 735743.

[19] Duprey, S., Naaim, A., Moissenet, F., Begon, M., and Chèze, L., 2017, "Kinematic Models of the Upper Limb Joints for Multibody Kinematics Optimisation: An Overview," J. Biomech., 62, pp. 87-94.

[20] Seth, A., Sherman, M., Eastman, P., and Delp, S., 2010, "Minimal Formulation of Joint Motion for Biomechanisms," Nonlinear Dyn., 62(1), pp. 291-303.

[21] Seth, A., Matias, R., Veloso, A. P., and Delp, S. L., 2016, "A Biomechanical Model of the Scapulothoracic Joint to Accurately Capture Scapular Kinematics during Shoulder Movements," PLOS ONE, 11(1), p. e0141028.

[22] Wu, G., van der Helm, F. C. T., Veeger, H. E. J. D., Makhsous, M., Van Roy, P., Anglin, C., Nagels, J., Karduna, A. R., McQuade, K., Wang, X., Werner, F. W., Buchholz, B., and International Society of Biomechanics, 2005, "ISB Recommendation on Definitions of Joint Coordinate Systems of Various Joints for the Reporting of Human Joint Motion--Part II: Shoulder, Elbow, Wrist and Hand," J. Biomech., 38(5), pp. 981-992.

[23] Shaheen, A. F., Alexander, C. M., and Bull, A. M. J., 2011, "Tracking the Scapula Using the Scapula Locator with and without Feedback from Pressure-Sensors: A Comparative Study," J. Biomech., 44(8), pp. 16331636.

[24] Lempereur, M., Brochard, S., Mao, L., and Rémy-Néris, O., 2012, "Validity and Reliability of Shoulder Kinematics in Typically Developing Children and Children with Hemiplegic Cerebral Palsy," J. Biomech., 45(11), pp. 2028-2034.

[25] Delp, S. L., Anderson, F. C., Arnold, A. S., Loan, P., Habib, A., John, C. T., Guendelman, E., and Thelen, D. G., 2007, "OpenSim: Open-Source Software to Create and Analyze Dynamic Simulations of Movement," IEEE Trans. Biomed. Eng., 54(11), pp. 1940-1950.

[26] Vasavada, A. N., Li, S., and Delp, S. L., 1998, "Influence of Muscle Morphometry and Moment Arms on the Moment-Generating Capacity of Human Neck Muscles," Spine, 23(4), pp. 412-422.

[27] Netter, F., 2011, Atlas d'anatomie humaine, Elsevier Masson, Philadelphia. 
[28] Söderkvist, I., and Wedin, P. A., 1993, "Determining the Movements of the Skeleton Using Well-Configured Markers," J. Biomech., 26(12), pp. 1473-1477.

[29] Legnani, G., Casolo, F., Righettini, P., and Zappa, B., 1996, "A Homogeneous Matrix Approach to 3D Kinematics and Dynamics - I. Theory," Mech. Mach. Theory, 31(5), pp. 573-587.

[30] Koontz, A. M., Cooper, R. A., Boninger, M. L., Souza, A. L., and Fay, B. T., 2004, "Scapular Range of Motion in a Quasi-Wheelchair Push," Int. J. Ind. Ergon., 33(3), pp. 237-248.

[31] Slavens, B. A., Graf, A., Krzak, J., Vogel, L., and Harris, G. F., 2011, "Upper Extremity Wheelchair Kinematics in Children with Spinal Cord Injury," 2011 Annual International Conference of the IEEE Engineering in Medicine and Biology Society, pp. 8158-8161.

[32] Bourgain, M., Hybois, S., Thoreux, P., Rouillon, O., Rouch, P., and Sauret, C., 2018, "Effect of Shoulder Model Complexity in Upper-Body Kinematics Analysis of the Golf Swing," J. Biomech., 75, pp. 154-158.

[33] Blache, Y., and Begon, M., 2018, "Influence of Shoulder Kinematic Estimate on Joint and Muscle Mechanics Predicted by Musculoskeletal Model," IEEE Trans. Biomed. Eng., 65(4), pp. 715-722.

[34] Laitenberger, M., Raison, M., Périé, D., and Begon, M., 2014, "Refinement of the Upper Limb Joint Kinematics and Dynamics Using a Subject-Specific Closed-Loop Forearm Model," Multibody Syst. Dyn., 33(4), pp. 413438.

[35] Matsui, K., Shimada, K., and Andrew, P. D., 2006, "Deviation of Skin Marker from Bone Target during Movement of the Scapula," J. Orthop. Sci., 11(2), pp. 180-184.

[36] Duprey, S., Cheze, L., and Dumas, R., 2010, "Influence of Joint Constraints on Lower Limb Kinematics Estimation from Skin Markers Using Global Optimization," J. Biomech., 43(14), pp. 2858-2862.
[37] Habachi, A. E., Duprey, S., Chèze, L., and Dumas, R., 2013, "Global Sensitivity Analysis of the Kinematics Obtained with a Multi-Body Optimisation Using a Parallel Mechanism of the Shoulder," Comput. Methods Biomech. Biomed. Engin., 16(sup1), pp. 61-62.

[38] Hybois, S., Lombart, A., Puchaud, P., Bascou, J., Lavaste, F., Pillet, H., and Sauret, C., 2017, "Effects of Ellipsoid Parameters on Scapula Motion during Manual Wheelchair Propulsion Based on Multibody Kinematics Optimization. A Preliminary Study," Comput. Methods Biomech. Biomed. Engin., 20(sup1), pp. 107-108.

[39] Rankin, J. W., Kwarciak, A. M., Mark Richter, W., and Neptune, R. R., 2010, "The Influence of Altering Push Force Effectiveness on Upper Extremity Demand during Wheelchair Propulsion," J. Biomech., 43(14), pp. 2771-2779.

[40] Morrow, M. M., Rankin, J. W., Neptune, R. R., and Kaufman, K. R., 2014, "A Comparison of Static and Dynamic Optimization Muscle Force Predictions during Wheelchair Propulsion," J. Biomech., 47(14), pp. 34593465.

[41] Slowik, J. S., McNitt-Gray, J. L., Requejo, P. S., Mulroy, S. J., and Neptune, R. R., 2016, "Compensatory Strategies during Manual Wheelchair Propulsion in Response to Weakness in Individual Muscle Groups: A Simulation Study," Clin. Biomech., 33, pp. 34-41.

[42] Reinbolt, J. A., Schutte, J. F., Benjamin J.Fregly, Byung II Koh, Raphael T Hafka, Alan D George, and Kim H. Mitchell, 2005, "Determination of Patient-Specific MultiJoint Kinematic Models through Two-Level Optimization," J. Biomech., 38(3), pp. 621-626.

[43] Andersen, M. S., Damsgaard, M., and Rasmussen, J., 2009, "Kinematic Analysis of Over-Determinate Biomechanical Systems," Comput. Methods Biomech. Biomed. Engin., 12(4), pp. 371-384. 